\title{
Late-onset sepsis due to urinary tract infection in very preterm neonates is not uncommon
}

\author{
Alexander B. Mohseny ${ }^{1} \cdot$ Veerle van Velze $^{1} \cdot$ Sylke J. Steggerda ${ }^{1}$. \\ Vivianne E. H. J. Smits-Wintjens ${ }^{1}$ • Vincent Bekker ${ }^{2} \cdot$ Enrico Lopriore $^{1}$
}

Received: 28 January 2017 / Revised: 1 October 2017 / Accepted: 3 October 2017 / Published online: 23 October 2017

(C) The Author(s) 2017. This article is an open access publication

\begin{abstract}
Urinary tract infection (UTI) is a common cause of sepsis in infants. Premature infants hospitalized at a neonatal intensive care unit often have risk factors for infection. In this group, the risk of UTI is not clearly known, and guidelines for urine analysis are not unanimous. We aimed to identify the risk of UTI in premature infants with central lines, suspected of late-onset sepsis. We analyzed all 1402 infants admitted to our hospital between 2006 and 2014 with a gestational age less than 32 weeks. Six hundred sixty-two episodes of sepsis evaluations were found with an unknown source of infection based on clinical symptoms. In half of this group, urine analysis was performed identifying UTI in 11.3\% (24/212). In 13 of these infants (54\%) with a UTI, infection was due to Candida albicans. In at least four episodes, the diagnosis
\end{abstract}

Communicated by Patrick Van Reempts

Enrico Lopriore

e.lopriore@lumc.nl

Alexander B. Mohseny

a.b.mohseny@lumc.nl

Veerle van Velze

veerlevanvelze@hotmail.com

Sylke J. Steggerda

s.j.steggerda@lumc.nl

Vivianne E. H. J. Smits-Wintjens

v.e.h.j.smits-wintjens@lumc.nl

Vincent Bekker

v.bekker@lumc.nl

1 Department of Neonatology, Willem-Alexander Children's Hospital, Leiden University Medical Center, Leiden, The Netherlands

2 Department of Pediatric Infectious Diseases, Willem-Alexander Children's Hospital, Leiden University Medical Center,

Leiden, The Netherlands and treatment would have been delayed if urine analysis had not been performed.

Conclusion: Based on these findings, we conclude that in premature infants with central lines, urine analysis should be performed routinely when signs of infection occur beyond $72 \mathrm{~h}$ after birth. Urine collection should not be delayed and cultures should preferably be performed before the start of the antibiotic treatment.

What is known:

- In preterm infants, the presence of other risk factors for infection might make clinicians reluctant to obtain urine cultures during sepsis evaluation.

- An internal survey demonstrated that there is no consensus within the NICUs in The Netherlands regarding urine analysis as part of LOS work-up.

What is new:

- The risk of UTI in the NICU population (11.3\%) is comparable to term infants; therefore, urine analysis should be performed routinely when LOS is suspected.

- Candida albicans was the most frequently (54\%) detected pathogen causing UTI in this population.

Keywords Urinary tract infection · Candida albicans . Late-onset sepsis $\cdot$ Premature infant $\cdot$ Central venous catheter
Abbreviations
BC Bag collection
CAD Catheter a demeure
EOS Early-onset sepsis
LOS Late-onset sepsis
NEC Necrotizing enterocolitis
NICU Neonatal intensive care unit
SA Suprapubic aspiration
SUC Sterile urethral catheterization 
UTI Urinary tract infection

VAP Ventilator-associated pneumonia

\section{Introduction}

Children admitted to the neonatal intensive care unit (NICU) are at increased risk of infections. Infection is an important determinant of length of stay, morbidity, and mortality. The risk of urinary tract infection (UTI) is reported $13.6-16.4 \%$ in term infants who present with fever or other signs of infection [1-3]. The overall occurrence of UTI is reported higher in preterm infants as compared to term infants [4]. In both groups, the incidence of UTI increases with postnatal age, varying from 1 to $2 \%$ in the first $72 \mathrm{~h}$ after birth up to $25 \%$ later on $[4,5]$. Therefore, routine early-onset sepsis (EOS) work-up in infants does not include urine cultures [5]. UTI in preterm infants may be secondary to bacteremia, but it may also start as primary infection in the urinary tract leading to bacteremia [6]. In both scenarios, diagnosing the UTI is of importance since infection may cause kidney damage or may be secondary to a congenital abnormality of the kidney and the urinary tract (CAKUT).

In preterm infants admitted at the NICU, data regarding urine cultures in the evaluation of late-onset sepsis (LOS) are limited [7]. Nevertheless, since the overall risk of UTI rises with decreasing gestational age, low birth weight, and postnatal age above $72 \mathrm{~h}$ after birth, urine should be analyzed when $\operatorname{LOS}$ is suspected $[4,5,7,8]$. However, the presence of other risk factors for infection might make clinicians reluctant to obtain urine cultures during the primary sepsis evaluation [9]. The use of central arterial or venous lines in these infants, providing a direct source of bacteremia, is such a risk factor and often the cause of blood stream infections [10]. This might suggest that a central line infection has a higher probability than a primary UTI to cause sepsis in these infants. Furthermore, presumed complications of sterile catheterization or suprapubic aspiration hamper urine collection and alternative collection methods such as bag collection have high rates of contamination [11]. An internal survey demonstrated that half of the NICUs in The Netherlands consistently include urine analysis as part of LOS work-up while the remaining centers only collect urine when additional UTI related symptoms are present. Therefore, rationale and recommendations are needed to either underline or discourage routine urine culturing when premature infants with central lines are evaluated for LOS.

The aim of this study was to evaluate the risk of UTI among premature infants with central lines who were evaluated for LOS. In addition, concordant blood cultures and ultrasonography images were analyzed to investigate the consequences of not (timely) diagnosing the UTI. Recorded data included side effects of sampling urine such as registered pain or complications, the treatment, and the presence of urinary tract malformations.

\section{Materials and methods}

\section{Study design}

We conducted a single center cohort study of all infants with a gestational age $<32$ weeks hospitalized at the NICU of the Leiden University Medical Center between 01 and 01-2006 and 01-01-2014 (Table 1). Infants with known congenital anomalies, in particular congenital anomalies of the kidney and urinary tract, were excluded from the study.

\section{Data collection}

Patient records of included infants were anonymously analyzed to identify any episodes of sepsis evaluation. Demographic details, urine and blood cultures, postnatal age at sepsis work-up (in hours after birth), presence of central lines, therapy of choice and duration, method of urine sampling registered complications of urine sampling, and ultrasonography results were collected for analysis.

\section{Definitions}

LOS was defined as clinical signs of sepsis as judged by the physician, developing more than $72 \mathrm{~h}$ after birth $[12,13]$. When an infant was evaluated for sepsis more than once, all new unrelated episodes, i.e., free of clinical sings or positive relevant cultures between two episodes, were included as separate events. Urine specimens were obtained by one of four methods: sterile urethral catheterization (SUC), suprapubic aspiration (SA), bag collection (BC), or collecting fresh urine from a catheter a demeure (CAD). Urine cultures with monocultural bacterial growth were considered positive based on the number of colony-forming units per milliliter (CFU/ml) dependent of the method of collection [14]. Central lines included umbilical arterial or venous lines and peripheral inserted central venous lines.

\section{Sepsis work-up and urine culture}

Sepsis work-up always included a blood culture prior to the start of antibiotic treatment. Culture of other specimens including urine and cerebral spinal fluid was advised but left to the discretion of the attending physician. Any bacterial growth from urine collected by SA was considered positive. Mono-cultural bacterial growth of $>10.000-100.000 \mathrm{CFU} / \mathrm{ml}$ collected by SUC or freshly collected CAD samples was defined as positive. Upon pathogenic growth from CAD urine, the catheter was removed and antibiotic treatment was 
Table 1 Patients' characteristics of the study cohort

\begin{tabular}{|c|c|c|}
\hline Patients & $N$ & \\
\hline Total analyzed & 1402 & \\
\hline $\begin{array}{l}\text { Congenital abnormalities } \\
\text { excluded }\end{array}$ & 86 & \\
\hline Sepsis episodes analyzed & $\begin{array}{l}1548 \text { (from } 1316 \\
\text { patients) }\end{array}$ & \\
\hline Episodes excluded & 1113 & \\
\hline Episodes included ${ }^{i i i}$ & 435 (from 389 patients) & \\
\hline \multirow[t]{2}{*}{ Gestational age } & Range (weeks) & Mean \\
\hline & $23-31$ & 27.7 \\
\hline \multirow[t]{2}{*}{ Birth weight } & Range (grams) & Mean \\
\hline & $430-2040$ & 1044 \\
\hline Gender & $N$ & Ratio \\
\hline Male & 216 & $1.25: 1$ \\
\hline Female & 173 & \\
\hline Central catheters ${ }^{\text {iv }}$ & $N$ & \\
\hline Umbilical & 336 & \\
\hline Peripheral & 222 & \\
\hline \multirow[t]{2}{*}{ Time of sepsis evaluation } & Range (hours after birth) & Mean \\
\hline & $73-1781$ & 264 \\
\hline Urine cultures & $N$ & $\begin{array}{r}\text { Positiv } \\
\quad(\%)\end{array}$ \\
\hline Total & 212 (from 192 patients) & 11.3 \\
\hline Suprapubic aspiration & 18 & 5.6 \\
\hline Sterile urethral catheterization & 102 & 15.6 \\
\hline Catheter a demeure & 30 & 13.3 \\
\hline Bag collection & 56 & 5.3 \\
\hline
\end{tabular}

Different characteristics are grouped within the emphasized factor, such as patients, urine cultures etc

${ }^{i}$ Congenital abnormalities included syndromes associated with chromosomal anomalies, cyanotic cor vitia, congenital defects of an organ system including the urinary tract, hydrops fetalis, and proven metabolic disorder

${ }^{i i}$ The following sepsis evaluations were excluded:

- Pre/perinatal risk factors $(N=743)$ defined as unknown cause of the premature birth, infants with clinical signs of sepsis at birth, or a combination of two or more factors including maternal fever, maternal group B streptococcal (GBS) positivity without antibiotic prophylaxis or previous child with GBS sepsis, premature rupture of the membranes longer than $18 \mathrm{~h}$ before birth or asphyxia.

- No central line within $24 \mathrm{~h}$ of LOS evaluation $(N=81)$.

- $\operatorname{EOS}(N=146)$.

- Suspected NEC $(N=41)$.

- Suspected VAP $(N=42)$.

- Iatrogenic $(N=8)$ after reposition or manipulation of a central venous line.

- Unknown reason of the sepsis evaluation $(N=52)$.

iii Please note that this number of episodes exceeds the total number of infants included, since multiple episodes of sepsis were included when the episodes were unrelated

${ }^{\text {iv }}$ Some included infants had umbilical and peripheral central lines

initiated. Follow-up urine analysis was performed to control the efficacy of the treatment. Urine specimens from $\mathrm{BC}$ were only considered positive if there was mono-cultural growth $>100.000 \mathrm{CFU} / \mathrm{ml}$ of a urinary pathogen and the clinical course supported UTI.

\section{Statistical analysis}

The minimal needed size of the study population was based on the identification of UTI risk in preterm NICU-hospitalized infants with central lines. Reported data suggested UTI rates around $10 \%$ in infants without central lines [1,2, 4], and we defined that a risk rate of $2 \%$ or higher (since a risk of $1-2 \%$ is accepted in EOS) would justify routine urine analysis as part of LOS evaluation. The power calculation showed that at least 360 patients should be included to detect a difference rate of 2 to $10 \%$ with a power (beta) of 0.9 allowing an error rate (alpha) of 0.05. Data was analyzed by using IBM SPSS data analysis software version 22 .

\section{Results}

\section{Cohort population}

Between 2006 and 2014, 1402 newborns with a gestational age less than 32 weeks were admitted to our unit of which 1316 were included. Together, these infants had undergone 1548 episodes of unrelated sepsis evaluation. Next, all episodes with sepsis evaluation because of pre/perinatal infection risks or with a known or plausible origin outside the urinary tract such as necrotizing enterocolitis (NEC) or ventilatorassociated pneumonia (VAP) were excluded, resulting into 662 remaining episodes with sepsis of unknown origin. Of these episodes, 581 had central lines at the time of sepsis work-up, and 435 (from 389 neonates) were suspected of infection beyond $72 \mathrm{~h}$ after birth. In this group, urine was analyzed in 212 episodes (from 192 neonates, 49\%, Table 1).

\section{Number of UTI}

Out of 212 urine cultures, a total of 24 (11\%) showed pathogenic growth. Thirteen times (54\%) Candida albicans was cultured, three times (13\%) Escherichia coli, three times (13\%) Staphylococcus aureus, one (4\%) Morganella morganii, one Klebsiella pneumoniae, one Enterobacter cloacae, one Klebsiella oxytoca, and one Streptococcus agalactiae (Table 2).

\section{Method of urine collection}

Urine was collected from 102 infants by SUC (48\%), 56 times by $\mathrm{BC}(26 \%), \mathrm{CAD}$ in 30 episodes (14\%), and SA in 18 episodes (9\%). Of the six remaining episodes (3\%), specifics about the method of collection could not be tracked. Side effects such as pain or complications after urine collection 
Table 2 Overview of the pathogens that were cultured from urine analysis and related characteristics

\begin{tabular}{|c|c|c|c|c|c|}
\hline Gestational age (weeks) & $\begin{array}{l}\text { Time of evaluation } \\
\text { (hours after birth) }\end{array}$ & Pathogen & Urine collection method & $\begin{array}{l}\text { Ultrasonography } \\
\text { of the urinary tract }\end{array}$ & $\begin{array}{l}\text { Concordant } \\
\text { growth in other cultures }\end{array}$ \\
\hline 23 & 1200 & Candida albicans & $\mathrm{BC}$ & Normal & Skin \\
\hline 24 & 137 & Candida albicans & SUC & Not performed & Skin and blood \\
\hline 24 & 239 & Candida albicans & SUC & Normal & Blood \\
\hline 24 & 279 & Candida albicans & SA & Normal & Skin and blood \\
\hline 25 & 133 & Candida albicans & $\mathrm{BC}$ & Not performed & Skin \\
\hline 26 & 172 & Candida albicans & SUC & Normal & Pharynx \\
\hline 26 & 88 & Candida albicans & SUC & Normal & Blood \\
\hline 26 & 82 & Candida albicans & SUC & Fungus balls & Blood and CSF \\
\hline 26 & 344 & Candida albicans & SUC & Normal & Sputum and blood \\
\hline 26 & 301 & Candida albicans & SUC & Normal & Blood \\
\hline 27 & 416 & Escherchia coli & CAD & Not performed & Eye fluid \\
\hline 27 & 864 & Klebsiella oxytoca & SUC & Normal & No \\
\hline 27 & 278 & Candida albicans & SUC & Normal & No \\
\hline 28 & 281 & Candida albicans & SUC & Normal & No \\
\hline 28 & 840 & Klebsiella pneumoniae & $\mathrm{BC}$ & Not performed & No \\
\hline 28 & 194 & Escherchia coli & SUC & Not performed & No \\
\hline 28 & 125 & Staphylococcus aureus & SUC & Not performed & Blood \\
\hline 29 & 651 & Enterobacter cloacae & CAD & Not performed & Sputum \\
\hline 29 & 627 & Escherchia coli & CAD & Normal & No \\
\hline 29 & 195 & Staphylococcus aureus & CAD & Not performed & Blood \\
\hline 29 & 272 & Candida albicans & SUC & Normal & No \\
\hline 29 & 452 & Streptococcus agalactiae & SUC & Not performed & Blood \\
\hline 30 & 310 & Morganella morganii & SUC & Normal & Blood \\
\hline 30 & 93 & Staphylococcus aureus & SUC & Not performed & Blood \\
\hline
\end{tabular}

$C S F$ cerebrospinal fluid, $S U C$ sterile urethral catheterization, $S A$ suprapubic aspiration, $B C$ bag collection, $C A D$ catheter a demeure (CAD)

were not reported. From the 24 positive urine cultures, 16 were collected by SUC (67\%), 4 by CAD (17\%), 3 by BC $(12 \%)$, and 1 by SA (4\%).

\section{Ultrasonography}

To detect any (possibly missed) urinary tract abnormalities related to UTI, reports of all performed ultrasonography examination were analyzed. Within the total group of 1402 infants, ultrasonography of the urinary tract was performed 138 times (9\%). In 20 of these studies, abnormalities were found (14\%). These 20 abnormal outcomes were originating from 16 infants; as in some infants, imaging of the urinary tract was repeated for follow-up. The abnormalities included abnormal size for the age (3), hydronephrosis unrelated to infection (7), nephrocalcinosis (1), renal vein thrombosis (1), extra renal pyelum (2), and fungus balls in two cases. No abnormalities predisposing for UTI were found and only the two cases with fungus balls were interpreted as complications of UTI. Within the group with positive pathogen growth from the urine culture (24 episodes), 14 ultrasound examinations were performed and showed an abnormality in only one infant; renal pelvis fungus balls in both kidneys. So, one infant was found with reported fungus balls in one kidney without pathogenic growth from urine and blood cultures. This infant was referred to our NICU after Candida UTI was diagnosed, and systemic treatment was initiated elsewhere explaining the negative cultures at our NICU.

\section{Discussion}

This study was designed to investigate the risk of UTI in NICUadmitted premature infants (gestational age $<32$ weeks) with central lines who were suspected of LOS. We found a high risk $(11.3 \%)$ of UTI in this specific population. Our data show that UTI is not uncommon and that the source of infection in 1 out of 10 episodes is the urinary tract. Interestingly, in these neonates, only in $40 \%$ urine was cultured. Prior to this study, we hypothesized that this was related to the assumption of clinicians that UTI is rare in this population because the presence of central lines is a more likely sepsis risk factor. Moreover, lack 
of a rigid and evidence-based protocol for this group of infants, such as available protocols for term neonates [14], might explain the motivation of the clinician not to prioritize urine analysis.

In addition, urine collection in premature infants by catheterization or suprapubic aspiration might be challenging. To overcome this, often, bag collection was used to screen the urine within the first sepsis evaluation and often repeated by an alternative method for a clean catch to culture when the screening indicated possible infection or contamination. However, repeating the collection results in a delay during which the patient most often has received antibiotics, which might cause false negative urine cultures. Alternative methods such as bladder stimulation and paravertebral lumbar massage techniques are reported with high rates of contamination $[11,15]$. In this cohort, UTI was found in three infants by using the method of bag collection showing monocultural growth without any sings of contamination. Although the possibility of contamination is not excluded, only three out of $56 \mathrm{BC}$ urine cultures $(5 \%)$ were considered positive. Moreover, based on these positive cultures, infants were treated with systemic therapy, and following urine cultures after treatment were sterile.

UTI was found in 24 infants, more than half $(54 \%, n=13)$ caused by Candida albicans who received systemic treatment. Candidiasis is known as a frequent cause of infection in NICU-admitted patients [16-19] and is related with a high mortality. From the 13 infants suffering from Candida albicans infection, most also had positive Candida growth from other cultures such as blood and skin. However, in six episodes, Candida was not detected in blood cultures, and concordant growth in skin and/or saliva cultures was only detected in three episodes, indicating that the Candida infection might have been missed, and the treatment might have been delayed if the urine cultures were not performed. So, in these patients, urine analysis was of crucial importance for their treatment and prognosis.

During the study period, fluconazole prophylaxis to reduce the risk of Candida infection was not routinely used at our NICU. Since the introduction of routine fluconazole prophylaxis in 2013, in premature infants with a gestational age $<27$ weeks and/or birth weight $<750 \mathrm{~g}$, only one case of Candida UTI was detected (positive urine cultures obtained by SUC). This might indicate that strict fluconazole prophylaxis might result in less UTI due to candida infections. However, urine analysis as part of LOS evaluation remains of crucial importance (in this case blood and surface cultures showed no pathogenic growth). Prospective studies in cohorts with fluconazole prophylaxis are needed to investigate the frequency of UTI in this population.

By conducting this cohort study, we conclude the following:
1. The risk of UTI in the studied population $(11.3 \%)$ is comparable to term infants, this might guide clinical treatment and have implications for follow-up.

2. Urine analysis should, therefore, be performed routinely when LOS is suspected in preterm neonates, also in the presence of central lines as the presumed risk factor for infection.

3. There is no evidence for complications caused by urine collection independent of the method used, so collecting urine should not be delayed and preferably performed before the start of antibiotic treatment.

4. Prospective studies are needed to determine the incidence of UTI in the NICU population without UTI predisposing factors when fluconazole prophylaxis is administered to examine whether routine urine analysis would still be justified.

Authors' Contributions AB Mohseny, V van Velze and E Lopriore assembled study protocol, included data, and analyzed preliminary data. $\mathrm{AB}$ Mohseny and $\mathrm{E}$ Lopriore performed the final data analyses and contributed to the writing of the manuscript. S Steggerda, EHJ SmitsWintjens and E Lopriore provided patients' data, contributed to data interpretation and writing of the manuscript. V Bekker contributed to data interpretation and writing of the manuscript.

\section{Compliance with ethical standards}

Conflict of interest The authors declare that they have no conflicts of interest.

Ethical approval This article does not contain any studies with human participants or animals performed by any of the authors.

The authors declare that all patients' data were handled in a coded fashion according to the LUMC's and Dutch national ethical guidelines.

Open Access This article is distributed under the terms of the Creative Commons Attribution 4.0 International License (http:// creativecommons.org/licenses/by/4.0/), which permits unrestricted use, distribution, and reproduction in any medium, provided you give appropriate credit to the original author(s) and the source, provide a link to the Creative Commons license, and indicate if changes were made.

\section{References}

1. Bonadio W, Maida G (2014) Urinary tract infection in outpatient febrile infants younger than 30 days of age: a 10-year evaluation. Pediatr Infect Dis J 33(4):342-344. https://doi.org/10.1097/INF. 0000000000000110

2. Ismaili K, Lolin K, Damry N, Alexander M, Lepage P, Hall M (2011) Febrile urinary tract infections in 0- to 3-month-old infants: a prospective follow-up study. J Pediatr 158(1):91-94. https://doi. org/10.1016/j.jpeds.2010.06.053

3. Lin DS, Huang SH, Lin CC, Tung YC, Huang TT, Chiu NC, Koa HA, Hung HY, Hsu CH, Hsieh WS, Yang DI, Huang FY (2000) Urinary tract infection in febrile infants younger than eight weeks of age. Pediatrics 105(2):E20

4. Tamim MM, Alesseh H, Aziz H (2003) Analysis of the efficacy of urine culture as part of sepsis evaluation in the premature infant. 
Pediatr Infect Dis J 22(9):805-808. https://doi.org/10.1097/01.inf. 0000083822.31857.43

5. Riskin A, Toropine A, Bader D, Hemo M, Srugo I, Kugelman A (2013) Is it justified to include urine cultures in early ( $<72$ hours) neonatal sepsis evaluations of term and late preterm infants? Am J Perinatol 30(6):499-504. https://doi.org/10.1055/s-0032-1329180

6. Downey LC, Benjamin DK Jr, Clark RH, Watt KM, Hornik CP, Laughon MM, Cohen-Wolkowiez M, Smith PB (2013) Urinary tract infection concordance with positive blood and cerebrospinal fluid cultures in the neonatal intensive care unit. J Perinatol 33(4): 302-306. https://doi.org/10.1038/jp.2012.111

7. Eliakim A, Dolfin T, Korzets Z, Wolach B, Pomeranz A (1997) Urinary tract infection in premature infants: the role of imaging studies and prophylactic therapy. J Perinatol 17(4):305-308

8. Bauer S, Eliakim A, Pomeranz A, Regev R, Litmanovits I, Arnon S, Huri H, Dolfin T (2003) Urinary tract infection in very low birth weight preterm infants. Pediatr Infect Dis J 22(5):426-430. https:// doi.org/10.1097/01.inf.0000065690.64686.c9

9. Foglia EE, Lorch SA (2012) Clinical predictors of urinary tract infection in the neonatal intensive care unit. J Neonatal-Perinatal Med 5(4):327-333. https://doi.org/10.3233/NPM-1262812

10. Padula MA, Dewan ML, Shah SS, Padula AM, Srinivasan L, McGowan KL, Mahoney KR, Harris MC (2014) Risk factors associated with laboratory-confirmed bloodstream infections in a tertiary neonatal intensive care unit. Pediatr Infect Dis J 33(10):10271032. https://doi.org/10.1097/INF.0000000000000386

11. Altuntas N, Tayfur AC, Kocak M, Razi HC, Akkurt S (2015) Midstream clean-catch urine collection in newborns: a randomized controlled study. Eur J Pediatr 174(5):577-582. https://doi.org/10. 1007/s00431-014-2434-Z

12. Wynn JL, Wong HR, Shanley TP, Bizzarro MJ, Saiman L, Polin RA (2014) Time for a neonatal-specific consensus definition for sepsis.
Pediatr Crit Care Med 15(6):523-528. https://doi.org/10.1097/ PCC.0000000000000157

13. Lukacs SL, Schrag SJ (2012) Clinical sepsis in neonates and young infants, United States, 1988-2006. J Pediatr 160(6):960-965 e961. https://doi.org/10.1016/j.jpeds.2011.12.023

14. Subcommittee on Urinary Tract Infection SCoQI, Management, Roberts KB (2011) Urinary tract infection: clinical practice guideline for the diagnosis and management of the initial UTI in febrile infants and children 2 to 24 months. Pediatrics 128(3):595-610. https://doi.org/10.1542/peds.2011-1330

15. Herreros Fernandez ML, Gonzalez Merino N, Tagarro Garcia A, Perez Seoane B, de la Serna MM, Contreras Abad MT, Garcia-Pose A (2013) A new technique for fast and safe collection of urine in newborns. Arch Dis Child 98(1):27-29. https://doi.org/10.1136/ archdischild-2012-301872

16. Nowell L, Moran C, Smith PB, Seed P, Alexander BD, Cotten CM, Wiener JS, Benjamin DK Jr (2010) Prevalence of renal anomalies after urinary tract infections in hospitalized infants less than 2 months of age. J Perinatol 30(4):281-285. https://doi.org/10.1038/ jp. 2009.147

17. Phillips JR, Karlowicz MG (1997) Prevalence of Candida species in hospital-acquired urinary tract infections in a neonatal intensive care unit. Pediatr Infect Dis J 16(2):190-194

18. Fridkin SK, Kaufman D, Edwards JR, Shetty S, Horan T (2006) Changing incidence of Candida bloodstream infections among NICU patients in the United States: 1995-2004. Pediatrics 117(5): 1680-1687. https://doi.org/10.1542/peds.2005-1996

19. Stoll BJ, Hansen N, Fanaroff AA, Wright LL, Carlo WA, Ehrenkranz RA, Lemons JA, Donovan EF, Stark AR, Tyson JE, Oh W, Bauer CR, Korones SB, Shankaran S, Laptook AR, Stevenson DK, Papile LA, Poole WK (2002) Late-onset sepsis in very low birth weight neonates: the experience of the NICHD Neonatal Research Network. Pediatrics 110(2 Pt 1):285-291 